\title{
Paclitaxel Alters Sensory Nerve Biomechanical Properties
}

2

\author{
Brian G. Bober ${ }^{1}$, Sameer B. Shah ${ }^{1,2}$
}

4

$5{ }^{1}$ Department of Bioengineering, University of California, San Diego, La Jolla, CA

$6{ }^{2}$ Department of Orthopaedic Surgery, University of California, San Diego, La Jolla, CA

7

$8 \quad$ * Address correspondence to:

99500 Gilman Drive, MC 0863

10 La Jolla, CA 92093

11 (858) 822-0720 (phone)

12 (858) 822-3807 (fax)

13 sbshah@ucsd.edu

14

15 Keywords: paclitaxel, biomechanics, nerve, modulus, microtubules

16

17 Abstract: Paclitaxel is an effective chemotherapeutic that, despite its common use, frequently

18 causes debilitating peripheral sensory neuropathy. Paclitaxel binds to and stabilizes

19 microtubules, and through unknown mechanisms, causes abnormal microtubule aggregation.

20 Given that microtubules contribute to the mechanical properties of cells, we tested the

21 hypothesis that paclitaxel treatment would alter the stiffness of sensory nerves. Rat sural

22 nerves were excised and soaked in Ringer's solution with or without paclitaxel. Nerves were

23 secured between a force transducer and actuator, and linearly strained. Stress-strain curves

24 were generated, from which tangent moduli and linear elastic moduli were calculated. Paclitaxel

25 treated nerves exhibited significantly higher moduli in both linear and transition regions of the

26 curve. A composite-tissue model was then generated to estimate the stiffness increase in the 
cellular fraction of the nerve following paclitaxel treatment. This model was supported experimentally by data on mechanical properties of sural nerves stripped of their epineurium, and area fractions of the cellular and connective tissue components of the rat sural nerve, calculated from immunohistochemical images. Model results revealed that the cellular components of the nerve must stiffen $12 x$ to $115 x$, depending on the initial axonal modulus assumed, in order to achieve the observed tissue level mechanical changes. Consistent with such an increase, electron microscopy showed increased microtubule aggregation and cytoskeletal packing, suggestive of a more cross-linked cytoskeleton. Overall, our data suggests that paclitaxel treatment induces increased microtubule bundling in axons, which leads to alterations in tissue-level mechanical properties.

\section{Word Count: 3,675}

\section{Introduction}

Paclitaxel is an effective and common chemotherapeutic that prevents cell division by stabilizing microtubules, thus preventing their depolymerization(Amos and Lowe,1999). Due to its systemic delivery, paclitaxel also accumulates in peripheral nerves, causing a debilitating sensory neuropathy(Argyriou et al.,2014). Because neurons are post-mitotic, mechanisms underlying paclitaxel-induced neuropathy must differ from those affecting dividing cells. Animal models of disease and patients display dying back of axons, reduced epidermal nerve fiber density(Boyette-Davis et al.,2011), demyelination(Argyriou et al.,2008), altered ion channel activity(Hara et al.,2013), and disruptions in axonal transport. The latter pathway is commonly hypothesized to underlie neuropathic progression; abnormal clustering of microtubules(Masurovsky et 
al.,1981; Masurovsky et al.,1983; Turner and Margolis,1984) is thought to impair delivery of vital proteins and organelles by microtubule-based motor complexes to distal axonal reaches.

Beyond their role in transport, microtubules also play important structural and biomechanical functions in axons(Garcia et al.,2012; Ouyang et al.,2013; Peter and Mofrad,2012), which may also be perturbed by paclitaxel. For example, a recent study demonstrated, using atomic force microscopy, that paclitaxel increases the compressive modulus of cell bodies in cultured dorsal root ganglia(Au et al.,2014). Paclitaxel also alters the response of central neurons to damage during high strain-rate loading(TangSchomer et al.,2010). Theoretical studies suggest that microtubules may influence tensile axonal biomechanics(Garcia, Pena et al.,2012; Peter and Mofrad,2012).

Whether and how structural and mechanical changes caused by paclitaxel influence biomechanics and physiological function at the whole nerve level has not been tested. Tensile loading is particularly relevant in peripheral nerves, which span articulating joints and can incur substantial regional strains(Aoki et al.,2005; Ochi et al.,2013; Topp and Boyd,2006). However, deformation beyond normal levels impairs action potential conduction(Wall et al.,1992). To protect neurons, strain is partially accommodated by axonal undulations, which straighten upon nerve deformation(Haninec,1986; Rydevik et al.,1990). In axons themselves, maintenance of structural integrity during axonal straightening and subsequent lengthening is likely to be modulated by the cytoskeleton, including microtubules. Increasing microtubule amount, length, stiffness, and bundling through paclitaxel treatment could alter the mechanical response and stability of this delicate biomechanical system, leading to 
73 impaired function. This study uses a pure sensory nerve model to test the hypothesis

74 that paclitaxel causes changes in the tensile properties of peripheral nerves. Our data

75 suggest that paclitaxel influences the structure and biomechanics of sensory nerves,

76 supporting a new hypothesis that altered neuronal biomechanics could contribute to

77 paclitaxel-induced sensory dysfunction.

78 Methods

79

\section{Mechanical testing}

12 to 20 week old Sprague-Dawley rats were sacrificed and sural nerves were exposed and freed of the surrounding connective tissue bilaterally. A $5 \mathrm{~mm}$ sural nerve segment just distal to the trifurcation point of the sciatic nerve was tied off with 6-0 nylon sutures in situ (fig 1a). Both nerve segments were excised and randomly assigned to be soaked in Ringer's solution (Aqueous solution of $\mathrm{NaCl}: 120 \mathrm{mM}, \mathrm{KCl}: 4 \mathrm{mM}, \mathrm{MgSO}_{4}$ :

$0.8 \mathrm{mM}, \mathrm{NaHCO}_{3}: 16 \mathrm{mM}$, glucose: $20 \mathrm{mM}$, and $\mathrm{CaCl}_{2}: 2.2 \mathrm{mM}$, brought to $\mathrm{pH} 7.4$ ) with or without the addition of $20 \mu \mathrm{M}$ paclitaxel for 2 hours. Each nerve segment was attached to an Aurora 402A force transducer and linear actuator and stretched in a chamber containing the appropriate Ringer's solution.

The length at which the nerve first incurred detectable passive load was recorded as $L_{0}$. Nerves were stretched in increments of $0.2-0.5 \mathrm{~mm}$ at a rate of $\sim 0.05 \mathrm{~mm} / \mathrm{sec}$ and held for $5 \mathrm{~min}$ before recording the force output, to allow stress relaxation as previously described(Luna et al.,2013; Shah and Lieber,2003). Because sutures securing the nerve to the force transducer and actuator were initially slack, nerve length was measured from suture to suture from images captured at each incremental deformation 
(fig 1b). All measurements were made blinded as to paclitaxel treatment. We alternately

96 normalized lengths to the length at which nerve stress was equal to $2 \mathrm{~g}$-force $/ \mathrm{mm}^{2}\left(L_{\sigma=2}\right)$.

97 Cross-sectional area was calculated from nerve diameters measured from images

98 captured at $L_{0}$, assuming the nerve as a cylinder. Engineering stress-strain $(\sigma-\varepsilon)$ curves were then generated, normalizing to $L_{0}$ or $L_{\sigma=2}$. Segmental strains were also measured using ink dots along the nerve, to determine the eventual region of failure.

To test the mechanical properties of nerves without their epineurium, sural nerves were excised as above. The tip of the inner sheath, which extruded beyond the 103 epineurial boundary(Walbeehm et al.,2004), was held with forceps and the epineurium was manually peeled away. Mechanical testing was performed as above.

The elastic modulus of each curve was calculated as the slope of the linear 106 region of each stress-strain curve at high strains. To compare tangent moduli at specific lower strains preceding the linear region, each stress-strain curve, with strain normalized to $L_{\sigma=2}$, was plotted from $\varepsilon=0$ to the start of the linear region, and fit with an exponential function $\sigma=A^{*} \exp \left(B^{*} \varepsilon\right)$ as for other biological tissues(Xiong et al.,2008; Yamanari et al.,2012). $A=2$, such that $\sigma=2 \mathrm{~g}-$ force $/ \mathrm{mm}^{2}$, by definition, at $\varepsilon=0$. All curves

111 had $\mathrm{R}^{2}$ values $>$.95. Tangent moduli of non-linear regions of each curve were then

112 calculated by the derivative of the exponential curve fit with respect to strain. The strain 113 at which the tangent modulus of the exponential equation equaled the slope in the linear 114 region was denoted as $\varepsilon_{L}$. The region of the stress-strain curve with $\varepsilon<\varepsilon_{L}$ are referred to 115 as the transition region. 
Because our higher-resolution mechanical testing apparatus was not designed to

117 measure forces larger than 50 grams-force, we performed additional testing on a

118 separate apparatus to measure the ultimate stress of 5 pairs of sural nerves.

119 Contralateral pairs of $\sim 5 \mathrm{~mm}$ segments of sural nerves were bathed in Ringer's solution

120 with or without paclitaxel as above, mounted between a force transducer (Omega,

121 DFG55-5) and a linear actuator, and strained in increments of $0.5 \mathrm{~mm}$ every 30

122 seconds. Maximum force at failure was recorded, and ultimate stress was calculated by

123 dividing the force by the resting cross sectional area of the nerve.

124 Modeling

125 The linear portion of the nerve was modeled as a composite tissue comprised of 126 epineurium, inner sheath (perineurium and endoneurium) connective tissue, and a

127 cellular fraction, which were stretched in parallel. The total modulus was expressed as:

$$
E_{w}=A_{e} E_{e}+A_{\text {in }} E_{\text {in }}+A_{c} E_{c}(1)
$$

129 where $A_{i}$ and $E_{i}$ denote area fractions and modulus, respectively, and subscripts of $w, e$, 130 in, and $c$ refer to those properties of the whole nerve, epineurium, inner sheath 131 connective tissue, and cells, respectively. 134 from:

$$
E_{w}=A_{e} E_{e}+A_{i c} E_{i c}(2)
$$


136 where $A_{i}$ and $E_{i}$ denote area fractions and modulus, respectively, and subscripts of $w, e$,

137 and ic refer to those properties of the whole nerve, epineurium, and inner sheath

138 combined with cells, respectively.

139 Transmission electron microscopy

C57/BI6 mice were anesthetized and sural nerves were exposed bilaterally.

141 Smaller mouse nerves enabled us to obtain a more accurate and comprehensive

142 representation of the entire axonal population compared to rat nerves. Nerves were

143 surrounded by a hydrophobic barrier and immersed in Ringer's solution containing

$14420 \mu \mathrm{M}$ paclitaxel for $2 \mathrm{hr}$. Nerves were then fixed and imaged via electron microscopy. A

145 custom MATLAB script was used to determine the nearest neighbor distance between

146 microtubules and the microtubule density in images of 13 myelinated fibers from

147 paclitaxel-treated and control nerves. Significance between parameters was tested

148 using a Student's t-test $(\alpha<0.05)$.

Immunohistochemistry and Gomori's Trichrome.

$10 \mu \mathrm{M}$ frozen rat sural nerve cross sections were labeled using an anti-laminin

151 primary antibody (1:1000, Sigma Aldrich, L9393) for $1 \mathrm{hr}$ at room temperature and Alexa

152 Fluor 488 secondary antibody (1:400, Life Technologies) for $1 \mathrm{hr}$ at room temperature.

153 Images were captured on a Leica SP5 confocal microscope with appropriate filters.

154 Gomori trichrome staining was performed as previously described(Gomori,1950). 
Images of sural nerve cross sections from 3 nerves labeled for laminin were used to calculate the cellular and connective tissue area fractions of the sural nerve. A

160 randomly selected sub-region from an image of each nerve was cropped and manually segmented by tracing the inner area of the laminin rings in ImageJ (fig $4 b-d$ ). The

162 cumulative areas of the manually segmented regions were divided by the total area of 163 the cropped region, then normalized to dimensions of the whole nerve as measured by 164 trichrome staining (fig 4a).

\section{$\underline{\text { Results }}$}

Because paclitaxel primarily causes sensory neuropathy, we examined the sural nerve, a purely sensory nerve branch of the sciatic nerve. As for many tissues, stressstrain curves showed a toe region in which nerves incurred substantial strains under

169 minimal tension, followed by a transition region in which the nerve stiffened, and finally, 170 a linear region with a constant slope. Strain was normalized to two lengths, $L_{0}$, the

171 length at which the nerve first began to incur detectable passive load, and $L_{\sigma=2}$, the 172 length at which the nerve stress was equal to $2 \mathrm{~g}$-force $/ \mathrm{mm}^{2}$. This stress was the 173 minimum stress at which nerves visibly began to lengthen and achieved a consistent 174 signal to noise ratio. Representative plots for control and paclitaxel-treated samples are 175 shown (fig 2a and b), with the latter also illustrating piece-wise curve fits for paired 176 nerves. Raw stress-strain curves for all nerves are also provided (supplementary fig 1). We first probed paclitaxel-induced changes to the mechanical properties of the 178 peripheral nerves in the linear regions of stress-strain curves (fig $2 a, b) . \varepsilon_{L}$, the point 
179 defining the start of the linear region (fig $2 b)$, trended $(p=.115)$ towards a lower value in 180 paclitaxel-treated nerves compared to contralateral control nerves (fig $2 e$ ). Paclitaxeltreated nerves were significantly stiffer in their linear region, based on elastic moduli 182 calculated from stress-strain curves normalized both to $L_{0}$ and $L_{\sigma=2}(p<0.05$, fig $2 c, d)$. 183 We also examined the maximum stress in 5 separate pairs of nerves stretched to 184 failure. Most paclitaxel treated nerves showed a higher ultimate stress, however no 185 significant difference was observed, potentially due to one paclitaxel treated nerve 186 failing at a much lower stress than all other nerves (supplementary fig 2).

To examine biomechanical properties in a more physiologically relevant range of 188 strains (Topp and Boyd,2006), we then examined the transition region of the stressstrain curve, which preceded the linear region. Tangent moduli were calculated from the 190 exponential curve used to fit the stress-strain curve between $\varepsilon=0$ to $\varepsilon_{L}$, and were 191 significantly higher (stiffer) in paclitaxel treated nerves than contralateral control nerves 192 for low strains $(\varepsilon=1-5 \%)$.

To better understand how increased stiffness in individual cells could influence 194 biomechanics of the whole nerve, we developed a theoretical composite-tissue model 195 based on the relative moduli and cross-sectional area fractions of connective tissue and 196 cellular components. As expected, as visible by trichrome labeling, sural nerves 197 displayed, from outside in, clear epineurial, perineurial and endoneurial compartments 198 (fig 4a). To predict whether and by how much the modulus of the cellular fraction 199 increased after paclitaxel treatment, we required area fractions and moduli of the 200 connective tissue and cell components in untreated nerves. Trichrome labeling revealed 201 the epineurial cross-sectional area to be $24.7 \pm 2.7 \%(n=3)$ (fig 4a). To determine area 
202 fractions of the cell and connective tissue compartments of the inner sheath, we labeled

203 sural nerve sections with antibodies targeting laminin, which sharply demarcated

204 cellular boundaries (fig $4 b-d)$. The cellular area fraction of the nerve was $43.8 \pm 0.9 \%(n$

$205=3)$ and the inner sheath connective tissue fraction was $31.1 \pm 0.5 \%(n=3)$.

206 We next determined the modulus of the epineurial and inner sheath regions by

207 mechanically testing sural nerves stripped of their epineurium (figure 5a). Following

208 peeling, the inner sheath still appeared structurally intact (figure $5 b$ ), with expected

209 laminin localization and a visible perineurial boundary. Sural nerves lacking their

210 epineurium were $\sim 5 \mathrm{x}$ more compliant than intact nerves (elastic moduli: $1.6 \pm .9 \mathrm{MPa}$

211 vs. $7.8 \pm 2.2 \mathrm{MPa}, \mathrm{p}<0.001)$.

212 Finally, an estimated modulus of the cellular fraction was needed. To our

213 knowledge, mechanical properties of axons excised from peripheral nerves have not

214 been measured. However, several studies have estimated axonal stiffness in vitro or

215 through simulations(Bernal et al.,2007; Dennerll et al.,1989; Garcia, Pena et al.,2012).

216 We therefore solved our model using axonal moduli ranging from $100 \mathrm{kPa}$ to $1 \mathrm{MPa}$,

217 based on the range of reported values in these studies Assuming these moduli, the

218 moduli of the epineurium and inner sheath connective tissue fractions were calculated

219 (equations 1 and 2). For paclitaxel-treated nerves, the connective tissue moduli was

220 assumed to stayed constant (i.e., no microtubules in this compartment) while the cell

221 fraction modulus was varied. Upon incorporating the modulus of intact nerves following

222 paclitaxel treatment, the modulus of the cellular fraction after paclitaxel treatment was

223 calculated (fig 6a). Additionally, we calculated a stiffening factor (ration of paclitaxel-

224 treated to untreated cell fraction modulus), as a function of initial cellular fraction 
225 stiffness. Results show that the cellular fraction must stiffen $115 x$ to $12 x$, depending on 226 the initial axonal modulus used (fig 6b).

In an effort to determine a structural basis for biomechanical changes, we next sought to determine the extent by which cytoskeletal structures changed in response to paclitaxel. We therefore treated performed transmission electron microscopy on untreated and paclitaxel-treated rodent sural nerves in vivo. Microtubules, other cytoskeletal filaments, and their crosslinks have been suggested to impact cell mechanical properties(Ouyang, Nauman et al.,2013; Peter and Mofrad,2012). Because paclitaxel directly affects microtubules, we quantified their density and spacing with and without paclitaxel treatment in myelinated and unmyelinated axons. Paclitaxel treated axons showed frequent clusters of microtubules not present in control axons (fig 3 a-d) with highly consistent spacing between microtubules $(34.88 \pm 3.83 \mathrm{~nm}$ center to center distance, $n=10$ pairs), suggestive of a crosslink with a characteristic length. In addition 238 to inducing large microtubule clusters, overall microtubule density and spacing also changed after paclitaxel treatment. In both myelinated and unmyelinated axons, paclitaxel treatment caused a marked increase in the microtubule density of the entire axon $(p<.05)$ and decreased the nearest neighbor distance between microtubules

$242(p<.01, f i g 3 e)$. The cytoplasmic regions in the paclitaxel-treated nerves appeared 243 darker, with increased cytoskeletal density than the contralateral control. In addition, 244 some paclitaxel treated axons ( $\sim 9 \%)$ appeared to have compacted regions cytoplasm 245 excessively dense with structural proteins (fig $3 d$ ); such malformations were not 246 observed in the control sural nerve. Interestingly, though some cell bodies of non247 neuronal cells were interspersed within the extraneuronal space, no microtubules were 
248 observed in endoneurial or perineurial compartments, suggesting that these regions are 249 not appreciably changed with paclitaxel treatment. Collectively, these results indicate 250 that paclitaxel treatment increased microtubule, and possibly other cytoskeletal protein, 251 content and connectivity in the axonal shaft, providing a potential structural basis for the 252 observed mechanical changes.

253 Discussion

254 Mechanical properties of paclitaxel-treated sensory nerves

To our knowledge, mechanical testing of sensory nerves such as the sural nerve 256 has not yet been performed, despite sensory branches often spanning joints and being 257 used as autologous grafts. Interestingly, elastic moduli in rodent sural nerves appear to 258 be an over an order of magnitude greater than those of sciatic nerves(Borschel et 259 al.,2003) and slightly lower than those of the porcine laryngeal nerve(Alexander et 260 al.,2010) and rabbit tibial nerve(Kwan et al.,1992). This could reflect several reasons, 261 including fewer fascicles(Jeronimo et al.,2005), smaller neurons, and a thicker 262 epineurium relative to the total cross-sectional nerve area, leading to differences in the 263 relative contributions of intra-epineurial fractions to whole nerve properties. In addition, 264 while several studies have suggested that paclitaxel increases microtubule bundling in 265 rat sciatic nerves, few studies have analyzed sensory nerve structure after paclitaxel 266 treatment(Flatters and Bennett,2006; Sahenk et al.,1994), despite the fact that 267 paclitaxel primarily produces sensory neuropathies. 
270 study. Paclitaxel additionally modifies the tensile response of cultured DRG

271 axons(Dennerll, Lamoureux et al., 1989), and may increase tensile axonal stiffness,

272 though this observation was not discussed by the authors and only reflected a single

273 sample in each group (figures 4 and 11 in(Dennerll, Lamoureux et al.,1989)). Here we

274 show that cellular properties can influence the tensile properties of a nerve (fig 2). This

275 finding is consistent with a tensile load-bearing role for intact neurons in nerves of C.

276 elegans(Hammarlund et al.,2007), which is impaired by destabilizing the spectrin

277 cytoskeleton; however, it contrasts with previous assumptions that the cellular portion of

278 the nerve does not contribute significantly to the stiffness of nerves(Rydevik, Kwan et

279 al.,1990; Sunderland and Bradley,1961; Topp and Boyd,2006; Walbeehm, Afoke et 280 al.,2004).

When a nerve is stretched, connective tissue and axons, which initially undulate, 282 unravel and begin to accommodate loads(Rydevik, Kwan et al.,1990). Conventionally, 283 this loading is believed to be primarily borne by the connective tissue, especially the 284 perineurium(Topp and Boyd,2006; Walbeehm, Afoke et al.,2004), with axonal fibers 285 contributing minimally. This argument is primarily supported by two observations: (i) 286 Wallerian degenerated nerve stumps have similar mechanical properties to intact 287 nerves(Sunderland and Bradley,1961); and (ii) rabbit tibial nerves stretched past the 288 point of plastic deformation show ruptured perineurium without observation of nerve 289 fiber or epineurial damage(Rydevik, Kwan et al.,1990). However, conclusions based on 290 these observations could be an oversimplification. In the first case, Wallerian 291 degeneration takes weeks, and could alter the mechanical properties of nerves during 292 that time via extracellular and intracellular remodeling. In the second case, nerve fibers 
293 do not remain in a single image plane through their entire course and thus are often

294 observed as discontinuous in 2D images, making confirmation of fiber rupture difficult to

295 observe. Alternatively, axons could be plastically deformed due to cytoskeletal protein

296 breakage, as opposed to cell membrane rupture.

297 A limitation of our study is that our methods ignore the viscous response of the 298 tissue, which could provide important information regarding changes in nerve 299 properties. Furthermore, because we are unable to visualize the interior of the nerve 300 during stretch, we cannot reliability interpret the integrity of inner sheath tissue and cells 301 from measurements such as ultimate stress (supplementary fig 2). In addition, the 302 method of paclitaxel delivery to the nerve via local soaking is not a clinical delivery 303 mechanism. However, this approach precludes confounding effects on nerve stiffness 304 or intraneural pressure after systemic delivery, including inflammatory or fibrotic 305 influences. Finally, while the concentration of taxol used here $(20 \mu \mathrm{M})$ was larger than 306 peak concentrations measured in human subjects(Vasantha et al.,2011), several animal 307 models for paclitaxel-induced neuropathy use peak plasma paclitaxel concentrations 308 nearly an order of magnitude higher than those used here(Huehnchen et al.,2013; 309 Sparreboom et al.,1996). Thus, though a direct comparison is difficult, due to the 310 metabolism of systemically injected paclitaxel, the fact that electron microscopy showed 311 significant structural changes within 2 hours, and literature reports also show bundled 312 microtubules in sciatic nerves treated with paclitaxel(Roytta and Raine,1986) suggests 313 that our dosage is appropriate for modeling paclitaxel induced neuropathy. 
Structurally, mechanical changes correlate with increased microtubule bundling and density. These structural changes are in agreement with previous observations that paclitaxel increases cytoskeletal connectivity in homogenized brain tissue(Turner and Margolis,1984) and brine shrimp(Campbell et al.,1989). Microtubule bundles seen in paclitaxel-treated axons via electron microscopy showed remarkably uniform spacing with center-to-center distances of $34.88 \pm 3.83 \mathrm{~nm}$ (fig $3 b$ ). Given microtubule diameters of $\sim 25 \mathrm{~nm}$, our data suggest a potential cross-linking (bundling) protein of $\sim 10 \mathrm{~nm}$ in

323 length. This dimension is close to but slightly smaller than spacings expected for microtubule crosslinking proteins such as tau (20-27 nm(Rosenberg et al.,2008)) or dynein (consisting of a 13-15 nm diameter ring and a 10-15 nm length stalk(Reck-

326 Peterson et al.,2006)), suggesting other microtubule associated proteins may be 327 involved. Neurofilaments also appear to be more densely packed around microtubule 328 bundles (fig $3 b, d$ ), though their potential role in nerve mechanics remains to be 329 determined. Finally, the perineurium contains densely packed layers of cells(Thomas, 1963) - we cannot exclude the possibility that nerve stiffening was due to mechanical changes in the perineurial cells, though electron microscopy (fig 3 ) does not 332 indicate many microtubules in these cells(Thomas, 1963).

Because the cytoskeleton anchors the cell to the surrounding extracellular matrix, 334 a more networked cytoskeleton could also alter the way axons strain with respect to 335 their surroundings. Stiffer axons or altered adhesion between the cell and its 336 extracellular environment could alter the unraveling of undulating axons, or change the 337 relative extension of cells and their surrounding matrix under asymmetric loads (e.g., 
338 shear). These less intuitive possibilities could additionally amplify the mechanical effects

339 of paclitaxel.

340

341

342

343

344

345

346 347 likely to be engaged during tensile loading.

\section{Model parameters and predictions}

Our model of nerve mechanics relies on several parameters relating to the stiffness of connective tissue and cells within the nerve which have not yet been reported. Interestingly, though the perineurium is suggested to be the primary load bearing tissue in nerves(Rydevik, Kwan et al.,1990), peeling of the epineurium dramatically decreased nerve stiffness and tensile modulus (fig 5), indicating that the epineurium contributes significantly to the overall stiffness of the sural nerve, and is

In addition, values of the tensile modulus of the cellular fraction of untreated nerves were also necessary to determine the stiffening effects of paclitaxel. Several in vitro and in silico studies have been performed to measure or estimate the stiffness of axons. The Young's modulus of PC12 neurites grown in vitro has been experimentally measured to be $12 \pm 2 \mathrm{kPa}$ (Bernal, Pullarkat et al.,2007). However, PC12 neurites are 10 times more compliant than DRG axons(Dennerll, Lamoureux et al.,1989), and so this value was not used. Cultured DRG neurites show a wide range of diameters from .05 to $1.20 \mu \mathrm{m}$ (Windebank et al.,1985) with a median diameter of $0.24 \mu \mathrm{m}$; using data from figure 4 in(Dennerll, Lamoureux et al.,1989) and the median reported cultured DRG diameter of $0.24 \mu \mathrm{m}$, one can roughly estimate the modulus of a cultured DRG axon to be $\sim 90 \mathrm{kPa}$. Finally, axonal moduli have been determined via modeling to be $\sim 1$ MPa(Garcia, Pena et al.,2012). The authors reconcile this difference with the above 
360 papers by noting that in vitro studies use short axons where the tensile modulus is

361 calculated based on force measurements taken from the entirety of the axon; the

362 weakest point along the axon therefore dominates stiffness measurements. The axon

363 does not necessarily show a uniform distribution of microtubules along its length(Garcia,

364 Pena et al.,2012; Zhou et al.,2004) and therefore such in vitro studies may

365 underestimate stiffness. Our model shows that paclitaxel treated cells must stiffen

366 considerably $(\geq 12 x)$ to cause the observed results, suggesting that paclitaxel induced

367 changes to tensile mechanical properties of cells are dramatic.

368 Conclusions

Nerves necessarily experience substantial strains during joint movement, 370 including regional deformations of $20-30 \%$ (Aoki, Takasaki et al.,2005; Ochi, Horiuchi et

371 al.,2013; Topp and Boyd,2006). Our results suggest that paclitaxel stiffens sensory

372 nerves, consistent with morphological observations of increased microtubule packing

373 and density. If these changes alter the magnitude or distribution of strains along a nerve

374 or impair axonal unraveling, thus forcing axons to incur larger loads than normal, nerve

375 fibers could be damaged by simple daily movement. Though this idea is currently highly

376 speculative, altered nerve mechanics may thus influence neuropathic progression. Such

377 damage may impact cell function directly, due to microtubule breakage, such as that

378 observed in neurons loaded at high strain rates (Tang-Schomer et al.,2012), or

379 indirectly, by amplifying axonal transport defects(Bober et al.,2015). Given the

380 possibility of a mechanical contribution to neuropathy, the physical management of

381 patients treated with paclitaxel, including the development of effective post- 
382 chemotherapy immobilization or physical therapy protocols, warrants further

383 investigation.

\section{Acknowledgements}

385 We gratefully acknowledge imaging by Dr. Malcolm Wood at the Scripps Research

386 Institute electron microscopy core facility, and support from the National Science

387 Foundation (CBET1042522), U.S. Army Medical Research and Materiel Command 388 (W81XWH1010773), and the UCSD Academic Senate.

\section{References}

391 Alexander, M. J., J. M. Barkmeier-Kraemer and J. P. Vande Geest (2010). "Biomechanical properties of

392 recurrent laryngeal nerve in the piglet." Annals of Biomedical Engineering 38(8): 2553-2562.

393 Amos, L. A. and J. Lowe (1999). "How Taxol stabilises microtubule structure." Chemistry and Biology 394 6(3): R65-69.

395 Aoki, M., H. Takasaki, T. Muraki, E. Uchiyama, G. Murakami and T. Yamashita (2005). "Strain on the ulnar 396 nerve at the elbow and wrist during throwing motion." Journal of Bone and Joint Surgery (American 397 Volume) 87(11): 2508-2514.

398 Argyriou, A. A., M. Koltzenburg, P. Polychronopoulos, S. Papapetropoulos and H. P. Kalofonos (2008).

399 "Peripheral nerve damage associated with administration of taxanes in patients with cancer." Critical 400 Reviews in Oncology/Hematology 66(3): 218-228.

401 Argyriou, A. A., A. P. Kyritsis, T. Makatsoris and H. P. Kalofonos (2014). "Chemotherapy-induced 402 peripheral neuropathy in adults: a comprehensive update of the literature." Cancer Management and 403 Research 6: 135-147. 
Au, N. P., Y. Fang, N. Xi, K. W. Lai and C. H. Ma (2014). "Probing for chemotherapy-induced peripheral neuropathy in live dorsal root ganglion neurons with atomic force microscopy." Nanomedicine: Nanotechnology, Biology, and Medicine.

407 Bernal, R., P. A. Pullarkat and F. Melo (2007). "Mechanical properties of axons." Physical Review Letters 408 99(1): 018301.

409 Bober, B. G., E. Gutierrez, S. Plaxe, A. Groisman and S. B. Shah (2015). "Combinatorial Influences of 410 Paclitaxel and Strain on Axonal Transport." Experimental Neurology.

411 Borschel, G. H., K. F. Kia, W. M. Kuzon, Jr. and R. G. Dennis (2003). "Mechanical properties of acellular 412 peripheral nerve." Journal of Surgical Research 114(2): 133-139.

413 Boyette-Davis, J., W. Xin, H. Zhang and P. M. Dougherty (2011). "Intraepidermal nerve fiber loss

414 corresponds to the development of taxol-induced hyperalgesia and can be prevented by treatment with 415 minocycline." Pain 152(2): 308-313.

416 Campbell, E. J., S. A. MacKinlay and T. H. MacRae (1989). "Cross-linking of microtubules by microtubule417 associated proteins (MAPs) from the brine shrimp, Artemia." Journal of Cell Science 93 ( Pt 1): 29-39.

418 Dennerll, T. J., P. Lamoureux, R. E. Buxbaum and S. R. Heidemann (1989). "The cytomechanics of axonal 419 elongation and retraction." Journal of Cell Biology 109(6 Pt 1): 3073-3083.

420 Flatters, S. J. and G. J. Bennett (2006). "Studies of peripheral sensory nerves in paclitaxel-induced painful 421 peripheral neuropathy: evidence for mitochondrial dysfunction." Pain 122(3): 245-257.

422 Garcia, J. A., J. M. Pena, S. McHugh and A. Jerusalem (2012). "A Model of the Spatially Dependent 423 Mechanical Properties of the Axon During Its Growth." Cmes-Computer Modeling in Engineering \&

424 Sciences 87(5): 411-432.

425 Gomori, G. (1950). "A rapid one-step trichrome stain." American Journal of Clinical Pathology 20(7): 661426664. 
427 Hammarlund, M., E. M. Jorgensen and M. J. Bastiani (2007). "Axons break in animals lacking beta-

428 spectrin." Journal of Cell Biology 176(3): 269-275.

429 Haninec, P. (1986). "Undulating course of nerve fibres and bands of Fontana in peripheral nerves of the

430 rat." Anatomy and Embryology 174(3): 407-411.

431 Hara, T., T. Chiba, K. Abe, A. Makabe, S. Ikeno, K. Kawakami, I. Utsunomiya, T. Hama and K. Taguchi

432 (2013). "Effect of paclitaxel on transient receptor potential vanilloid 1 in rat dorsal root ganglion." Pain

433 154(6): 882-889.

434 Huehnchen, P., W. Boehmerle and M. Endres (2013). "Assessment of paclitaxel induced sensory

435 polyneuropathy with "Catwalk" automated gait analysis in mice." PloS One 8(10): e76772.

436 Jeronimo, A., C. A. Jeronimo, O. A. Rodrigues Filho, L. S. Sanada and V. P. Fazan (2005). "Microscopic

437 anatomy of the sural nerve in the postnatal developing rat: a longitudinal and lateral symmetry

438 study." Journal of Anatomy 206(1): 93-99.

439 Kwan, M. K., E. J. Wall, J. Massie and S. R. Garfin (1992). "Strain, stress and stretch of peripheral nerve.

440 Rabbit experiments in vitro and in vivo." Acta Orthopaedica Scandinavica 63(3): 267-272.

441 Luna, C., L. Detrick, S. B. Shah, A. H. Cohen and H. Aranda-Espinoza (2013). "Mechanical properties of the

442 lamprey spinal cord: uniaxial loading and physiological strain." Journal of Biomechanics 46(13): 2194-

4432200.

444 Masurovsky, E. B., E. R. Peterson, S. M. Crain and S. B. Horwitz (1981). "Microtubule arrays in taxol-

445 treated mouse dorsal root ganglion-spinal cord cultures." Brain Research 217(2): 392-398.

446 Masurovsky, E. B., E. R. Peterson, S. M. Crain and S. B. Horwitz (1983). "Morphological alterations in

447 dorsal root ganglion neurons and supporting cells of organotypic mouse spinal cord-ganglion cultures

448 exposed to taxol." Neuroscience 10(2): 491-509. 
Ochi, K., Y. Horiuchi, T. Nakamura, K. Sato, H. Arino and T. Koyanagi (2013). "Ulnar nerve strain at the elbow in patients with cubital tunnel syndrome: effect of simple decompression." Journal of Hand Surgery, European Volume 38(5): 474-480.

Ouyang, H., E. Nauman and R. Shi (2013). "Contribution of cytoskeletal elements to the axonal mechanical properties." Journal of Biological Engineering 7(1): 21.

Peter, S. J. and M. R. Mofrad (2012). "Computational modeling of axonal microtubule bundles under tension." Biophysical Journal 102(4): 749-757.

Reck-Peterson, S. L., A. Yildiz, A. P. Carter, A. Gennerich, N. Zhang and R. D. Vale (2006). "Single-molecule analysis of dynein processivity and stepping behavior." Cell 126(2): 335-348.

Rosenberg, K. J., J. L. Ross, H. E. Feinstein, S. C. Feinstein and J. Israelachvili (2008). "Complementary dimerization of microtubule-associated tau protein: Implications for microtubule bundling and taumediated pathogenesis." Proceedings of the National Academy of Sciences of the United States of America 105(21): 7445-7450.

Roytta, M. and C. S. Raine (1986). "Taxol-induced neuropathy: chronic effects of local injection." Journal of Neurocytology 15(4): 483-496.

Rydevik, B. L., M. K. Kwan, R. R. Myers, R. A. Brown, K. J. Triggs, S. L. Woo and S. R. Garfin (1990). "An in vitro mechanical and histological study of acute stretching on rabbit tibial nerve." Journal of

Orthopaedic Research 8(5): 694-701.

Sahenk, Z., R. Barohn, P. New and J. R. Mendell (1994). "Taxol neuropathy. Electrodiagnostic and sural nerve biopsy findings." Archives of Neurology 51(7): 726-729.

Shah, S. B. and R. L. Lieber (2003). "Simultaneous imaging and functional assessment of cytoskeletal protein connections in passively loaded single muscle cells." Journal of Histochemistry and

Cytochemistry 51(1): 19-29. 
472 Sparreboom, A., O. van Tellingen, W. J. Nooijen and J. H. Beijnen (1996). "Nonlinear pharmacokinetics of

473 paclitaxel in mice results from the pharmaceutical vehicle Cremophor EL." Cancer Research 56(9): 2112-

$474 \quad 2115$.

475 Sunderland, S. and K. C. Bradley (1961). "Stress-Strain Phenomena in Denervated Peripheral Nerve

476 Trunks." Brain 84(1): 125-\&.

477 Tang-Schomer, M. D., V. E. Johnson, P. W. Baas, W. Stewart and D. H. Smith (2012). "Partial interruption

478 of axonal transport due to microtubule breakage accounts for the formation of periodic varicosities after

479 traumatic axonal injury." Experimental Neurology 233(1): 364-372.

480 Tang-Schomer, M. D., A. R. Patel, P. W. Baas and D. H. Smith (2010). "Mechanical breaking of

481 microtubules in axons during dynamic stretch injury underlies delayed elasticity, microtubule

482 disassembly, and axon degeneration." FASEB Journal 24(5): 1401-1410.

483 Thomas, P. K. (1963). "The connective tissue of peripheral nerve: an electron microscope study." Journal

484 of Anatomy 97: 35-44.

485 Topp, K. S. and B. S. Boyd (2006). "Structure and biomechanics of peripheral nerves: nerve responses to 486 physical stresses and implications for physical therapist practice." Physical Therapy 86(1): 92-109.

487 Turner, P. F. and R. L. Margolis (1984). "Taxol-induced bundling of brain-derived microtubules." Journal 488 of Cell Biology 99(3): 940-946.

489 Vasantha, J., G. Kannan, T. Goud, T. Palani, R. Vanitha, R. Anitha and J. Priya (2011). "Pharmacokinetic 490 evaluation of Paclitaxel in South Indian cancer patients: a prospective study." Journal of Young

$491 \quad$ Pharmacists 3(4): 322-328.

492 Walbeehm, E. T., A. Afoke, T. de Wit, F. Holman, S. E. Hovius and R. A. Brown (2004). "Mechanical 493 functioning of peripheral nerves: linkage with the "mushrooming" effect." Cell and Tissue Research $494 \quad 316(1): 115-121$. 
Wall, E. J., J. B. Massie, M. K. Kwan, B. L. Rydevik, R. R. Myers and S. R. Garfin (1992). "Experimental stretch neuropathy. Changes in nerve conduction under tension." Journal of Bone and Joint Surgery (British Volume) 74(1): 126-129.

Windebank, A. J., P. Wood, R. P. Bunge and P. J. Dyck (1985). "Myelination determines the caliber of dorsal root ganglion neurons in culture." Journal of Neuroscience 5(6): 1563-1569.

Xiong, J., S. M. Wang, W. Zhou and J. G. Wu (2008). "Measurement and analysis of ultimate mechanical properties, stress-strain curve fit, and elastic modulus formula of human abdominal aortic aneurysm and nonaneurysmal abdominal aorta." Journal of Vascular Surgery 48(1): 189-195.

Yamanari, M., K. Ishii, S. Fukuda, Y. Lim, L. Duan, S. Makita, M. Miura, T. Oshika and Y. Yasuno (2012). "Optical rheology of porcine sclera by birefringence imaging." PloS One 7(9): e44026.

Zhou, F. Q., J. Zhou, S. Dedhar, Y. H. Wu and W. D. Snider (2004). "NGF-induced axon growth is mediated by localized inactivation of GSK-3beta and functions of the microtubule plus end binding protein APC." Neuron 42(6): 897-912.

\section{Figure Legends}

Figure 1 - Mechanical testing of sural nerves

A) Sural nerves were exposed in rat cadavers. A $\sim 5 \mathrm{~mm}$ segment of each nerve immediately distal to the trifurcation point of the sciatic nerve was sutured in situ with 6-0 nylon sutures. Sc and Su indicate the sciatic and sural nerves, respectively. Scale bar $=5 \mathrm{~mm}$. B) Each nerve was tied to a force transducer and a linear actuator, and progressively strained over time until visible nerve rupture (arrow). Ink dots along the nerve were used to monitor regional deformation. 
517 Figure 2 - Paclitaxel treatment alters whole nerve mechanical properties

Stress-Strain curves of contralateral nerve pairs soaked in Ringer's solution with and without paclitaxel were generated normalized to $L_{0}(\mathrm{~A})$ and $L_{\sigma=2}(\mathrm{~B})$. Each curve was fit with an exponential function from strain 0 to $\varepsilon_{L}$, the point at which the slope of the function was equal to the slope in the linear region (arrow). The slope of in the linear region was determined. C) In all cases, the tensile modulus of the paclitaxel treated nerve was greater than or equal to that of the contralateral control nerve. D) The mean tensile modulus of paclitaxel treated sural nerves is significantly $\left({ }^{*}, \mathrm{P}<.05\right)$ greater than that of the contralateral control nerves. E) $\varepsilon_{L}$ did not significantly differ between paclitaxel treated and control nerves. F) Tangent modulus calculations in the transition region reveal that paclitaxel significantly increases nerve stiffness $\left({ }^{*}\right.$, $\mathrm{P}<.05)$ even at low strains. Error bars indicate standard error of the mean.

529 Figure 3 - Paclitaxel causes bundling of microtubules in sural nerves

Sural nerves were exposed in anesthetized mice. One sural nerve was surrounded in a hydrophobic boundary and soaked in $20 \mu \mathrm{M}$ paclitaxel for 2 hours. Mice were sacrificing and paclitaxel treated and control nerves were then fixed for processing. A) TEM image of a myelinated axon within control sural nerves showing expected morphology with microtubules (arrows) scattered throughout the axon B) An axon within a paclitaxel -treated sural nerve shows a contraction of the cytoplasm and buildup of electron dense proteins indicated by the overall darker appearance. Microtubules form clusters of parallel arrays (boxes) and increase dramatically in number. A digitally zoomed inset is provided for increased clarity. C) Control sural 
nerve axons show intact myelin and axonal structure. D) After paclitaxel treatment, many axons show signs of myelin abnormalities and cytoskeletal collapse. E) Microtubules appear significantly closer together after paclitaxel treatment $\left({ }^{*}, \mathrm{P}<.05\right)$. F) Overall microtubule density per axon increases significantly after paclitaxel treatment $\left({ }^{*}, \mathrm{P}<.05\right)$. Error bars indicate standard error of the mean. Scale bars indicate $500 \mathrm{~nm}(\mathrm{~A}$ and $\mathrm{B})$ and $5 \mu \mathrm{m}(\mathrm{C}$ and $\mathrm{D})$.

\section{Figure 4 - Sural Nerve Histology}

A) Gomorri Trichrome stain of a rat sural nerve cross-section, used to calculate epineurial area. Traces indicate sample boundaries from which area fractions were calculated for the inner sheath and epineurial compartments. B) Cross-section of a rat sural nerve stained for laminin. C) In order to estimate the area fraction of the cellular components of the inner sheath, smaller regions of laminin staining were digitally cropped and the inner regions of the laminin rings were manually sectioned in ImageJ (D). Area of the inner rings of laminin relative to the entire cropped region was then computed. Scale bars indicate $25 \mu \mathrm{m}$. normalized to $L_{0}$. B) Cross-section of sural nerve stripped of epineurium labeled with a laminin antibody. Note that the nerve appears structurally intact. Scale bar indicates $100 \mu \mathrm{m}$.

559 Figure 6 - Model of paclitaxel induced stiffening of cells 
A) Our composite-tissue model was solved to determine the stiffness of the cell

561

fraction after paclitaxel treatment assuming a range of initial cell fraction moduli. B) After paclitaxel treatment, the cell fraction stiffens significantly. 

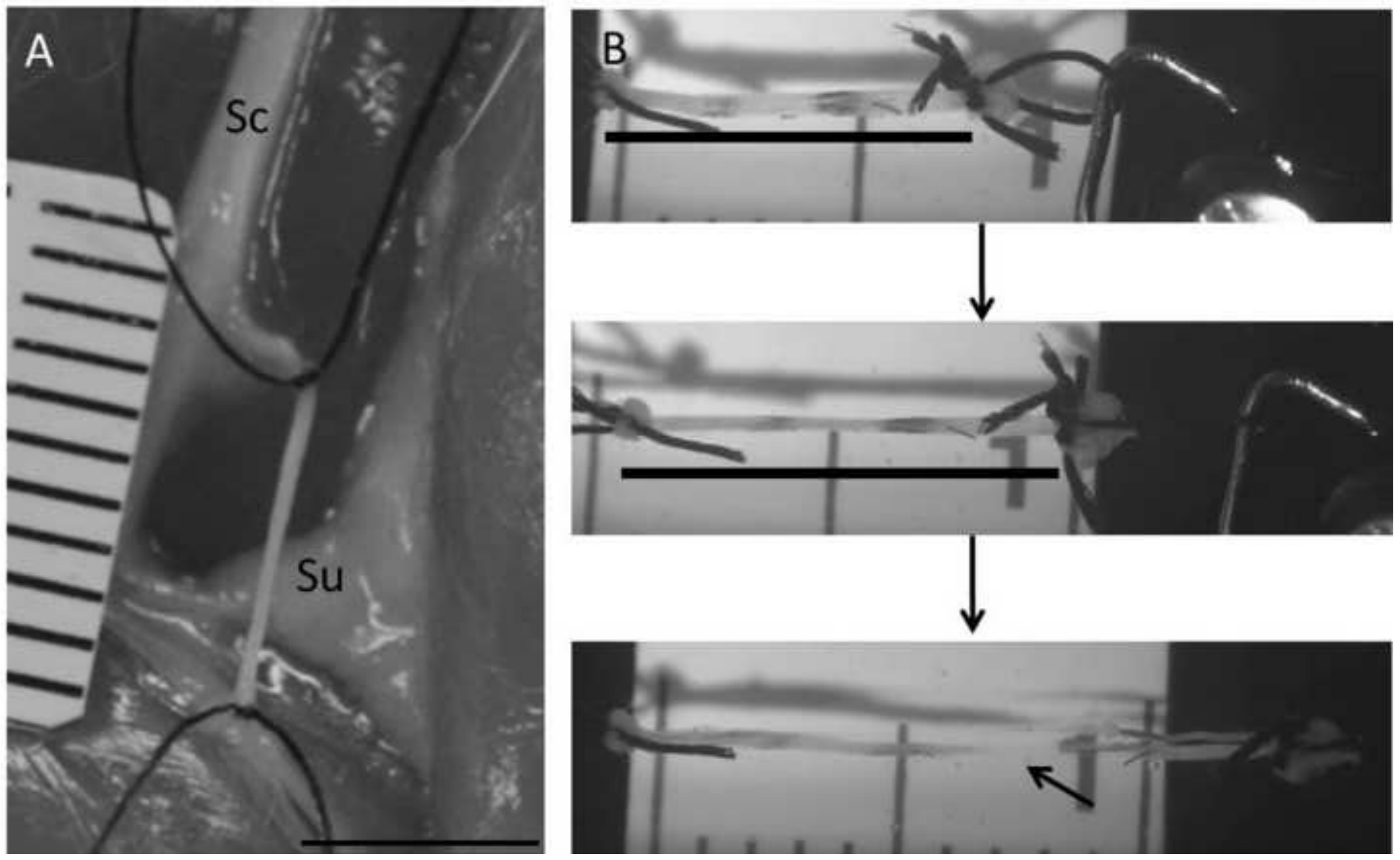


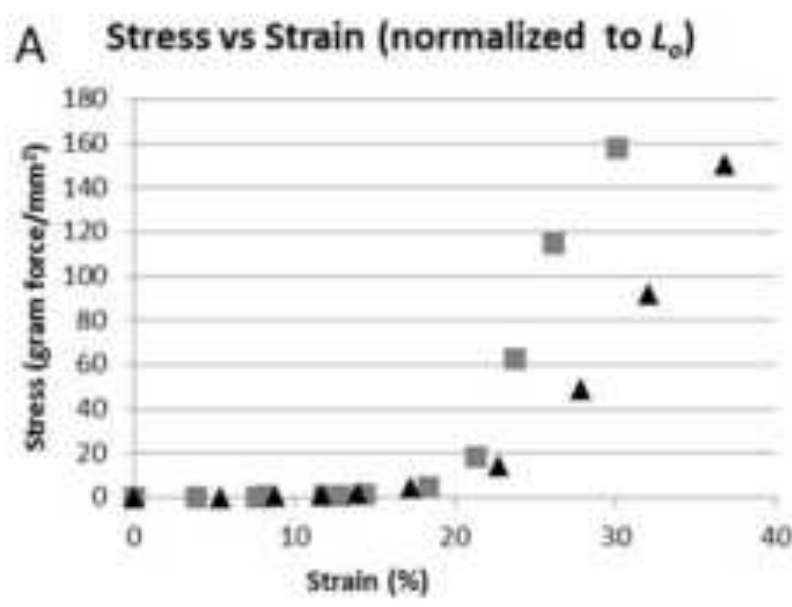

C Tensile Modulus (normalized to $L_{a=z}$ )

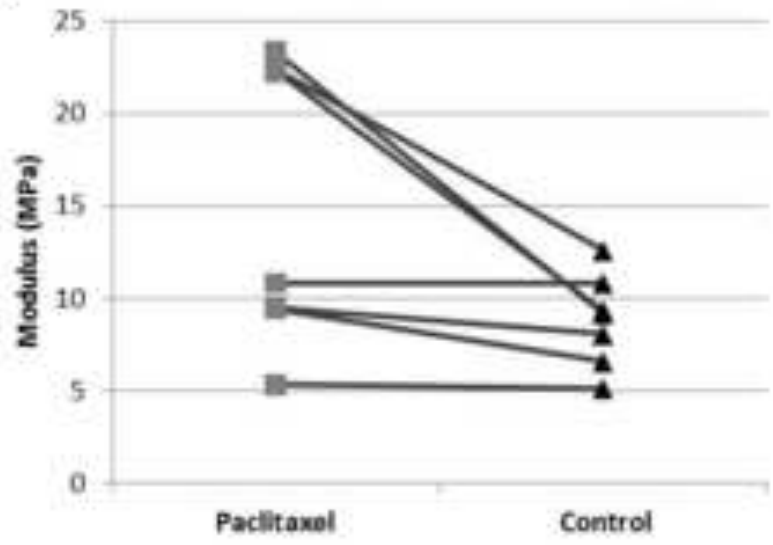

E

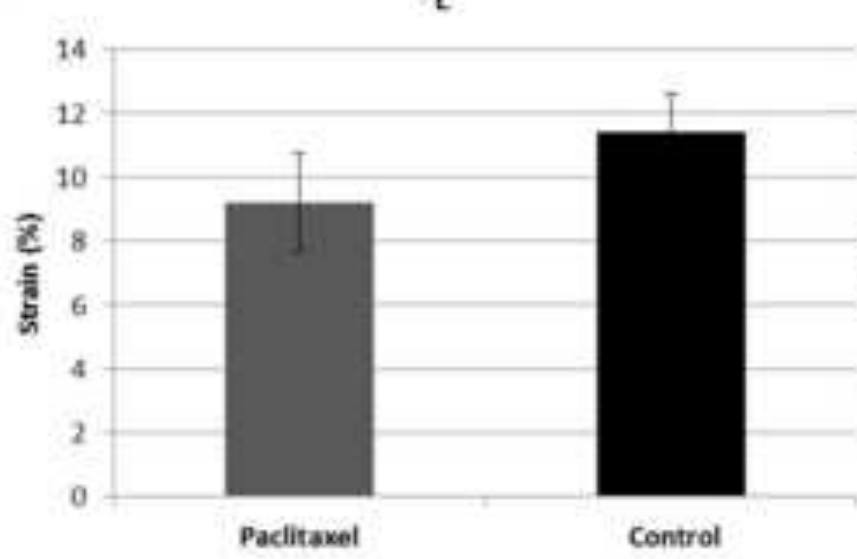

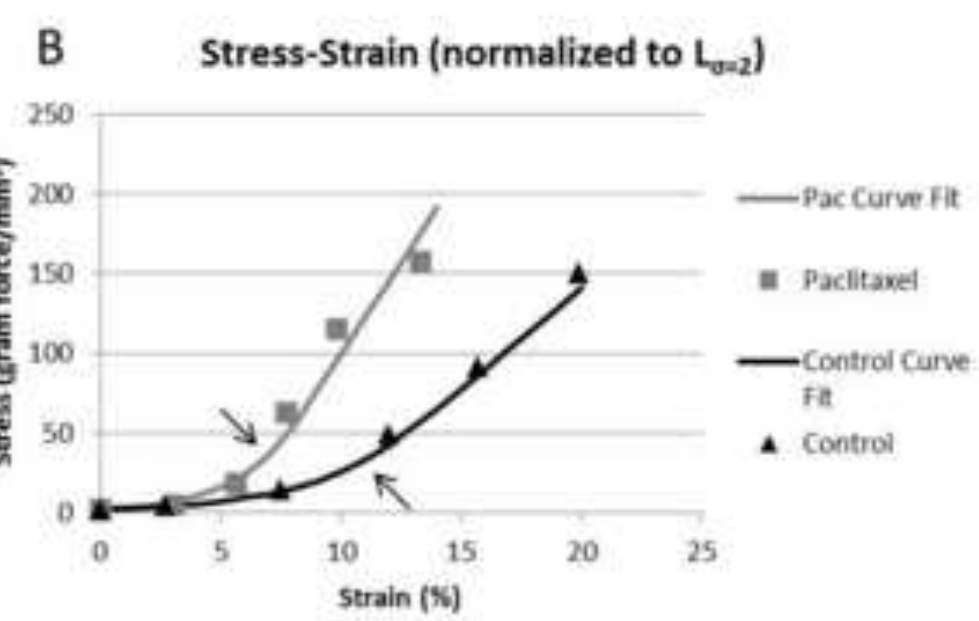

D

Tensile Modulus

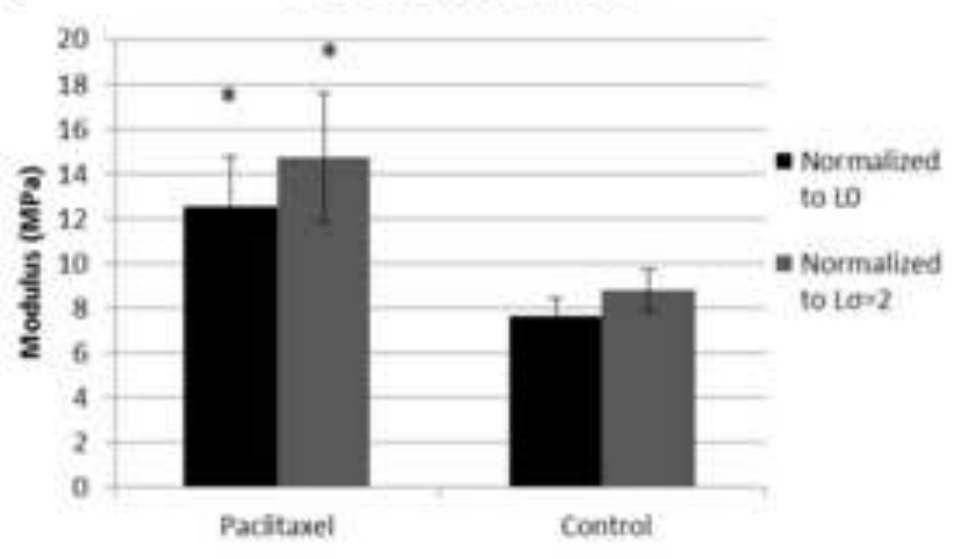

F

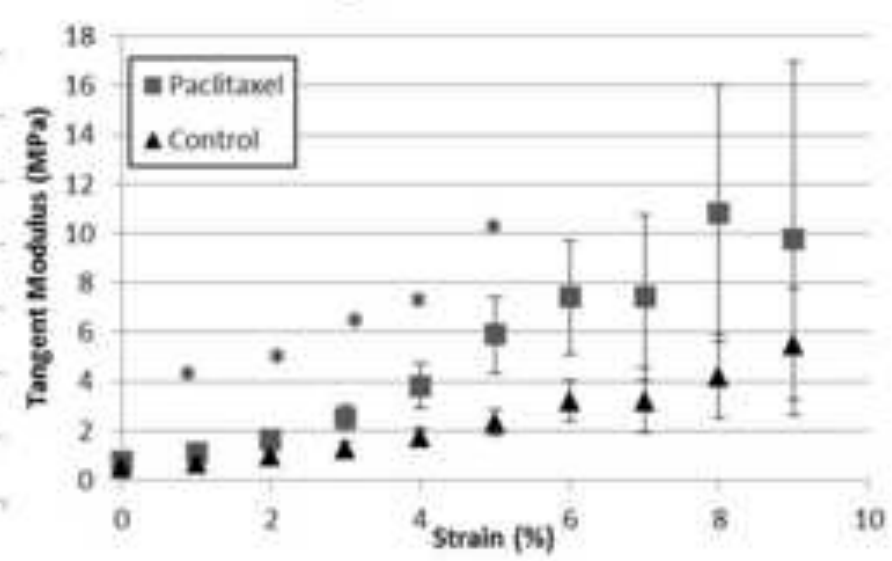



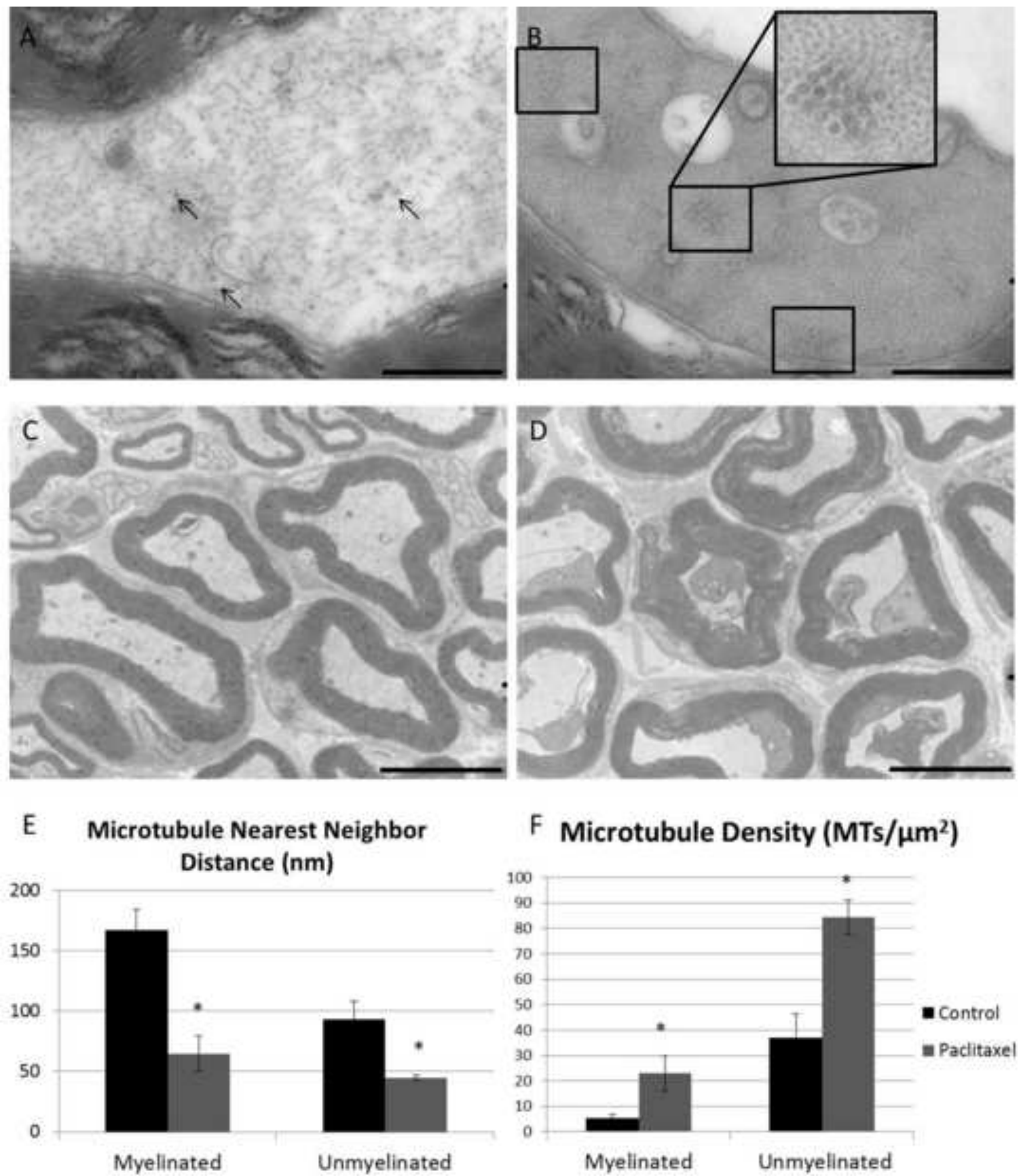

\section{F Microtubule Density (MTs/ $/ \mathrm{mm}^{2}$ )}

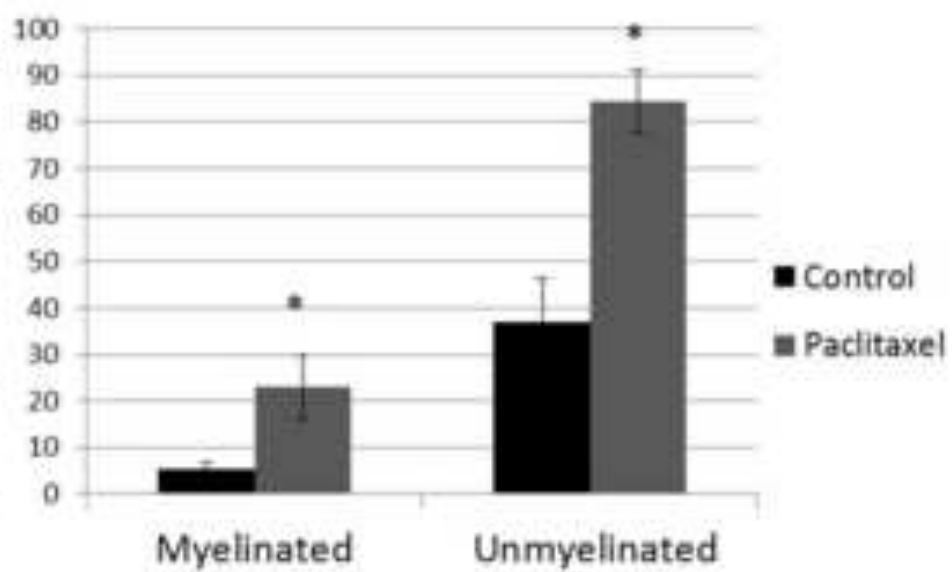



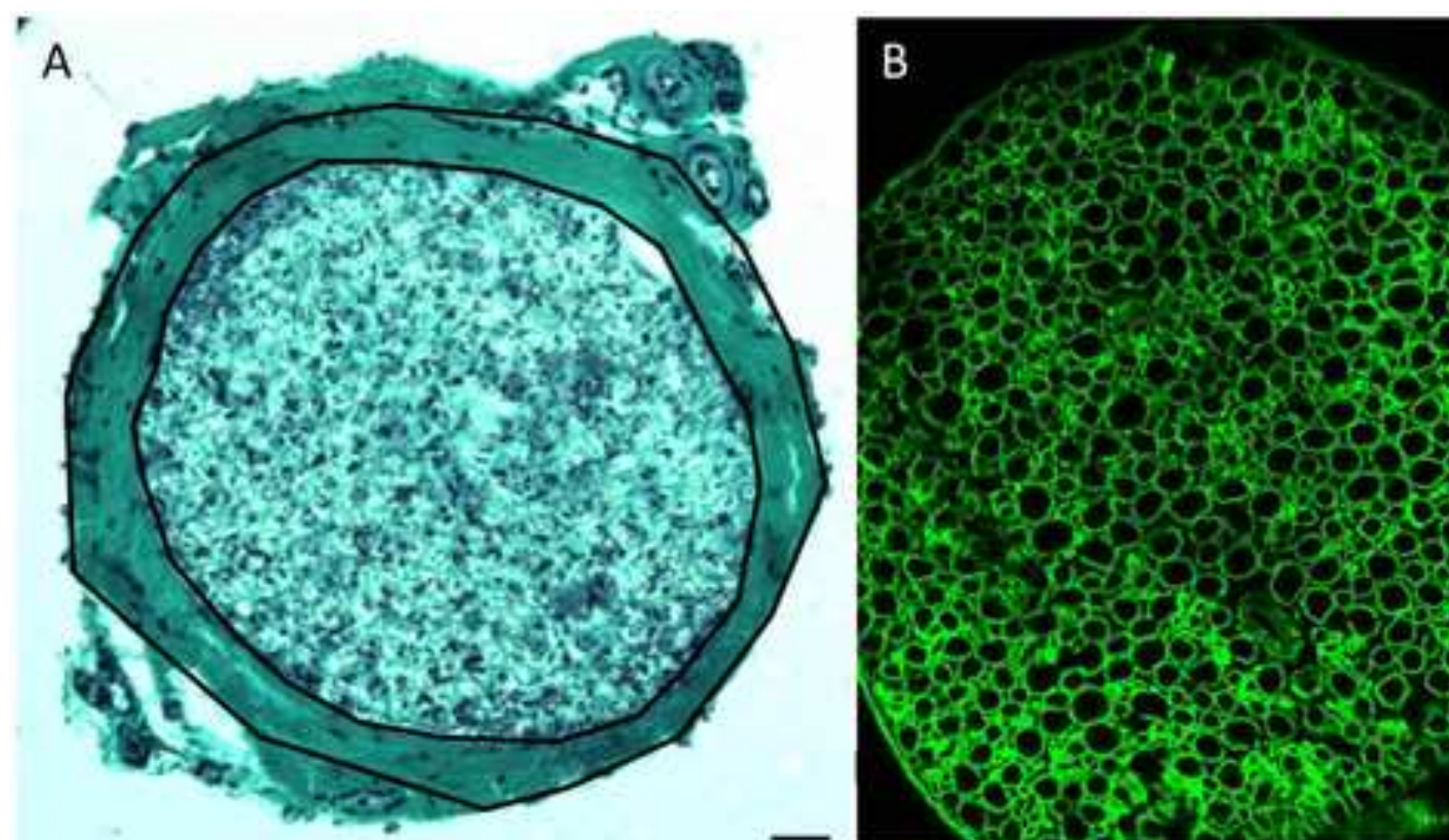

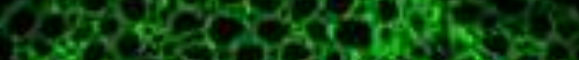

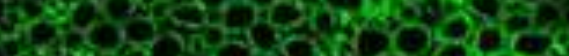

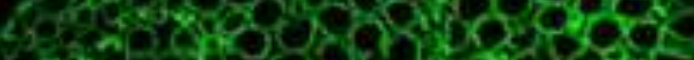

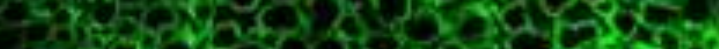

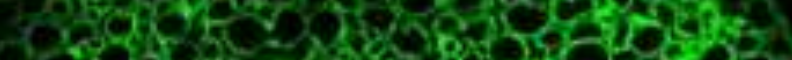

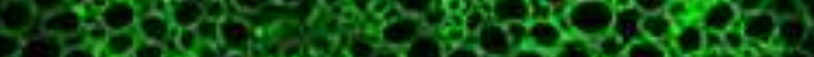

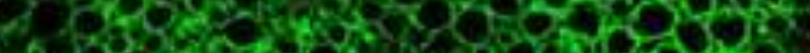

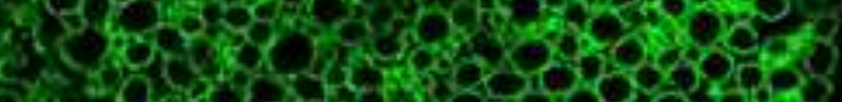

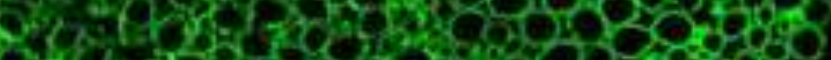

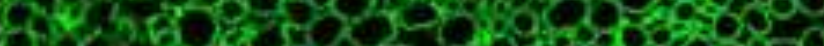

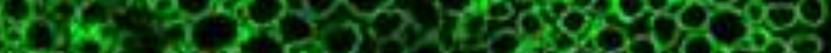

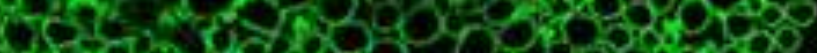

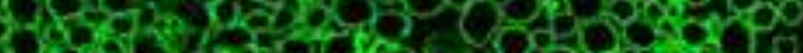

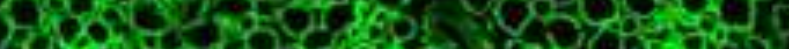

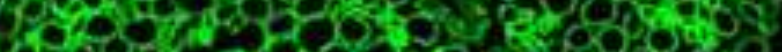

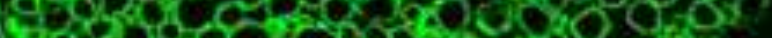

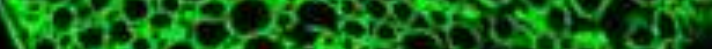

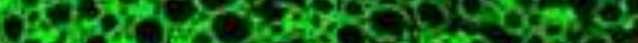
2 a 10 and Gowhosing

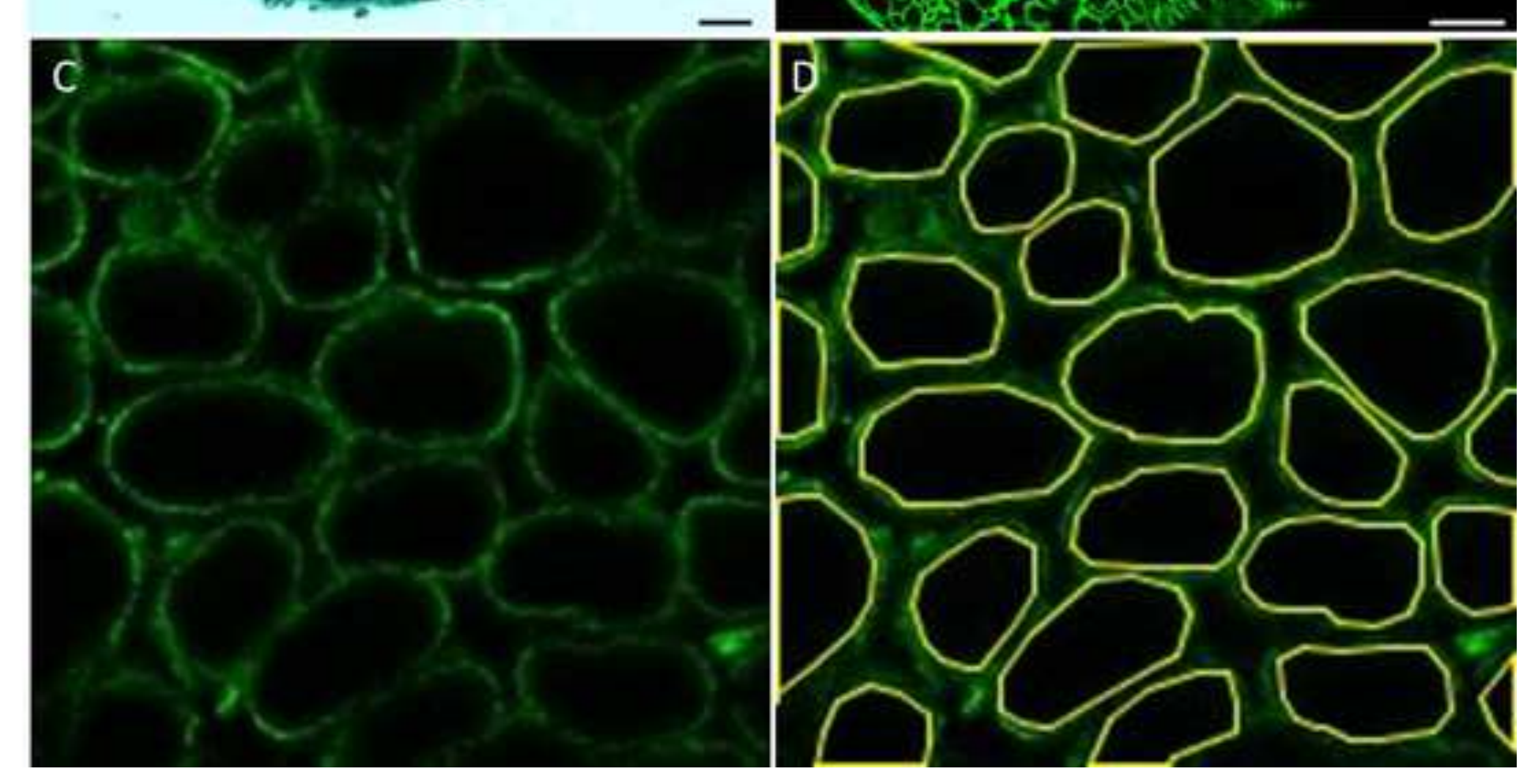


A

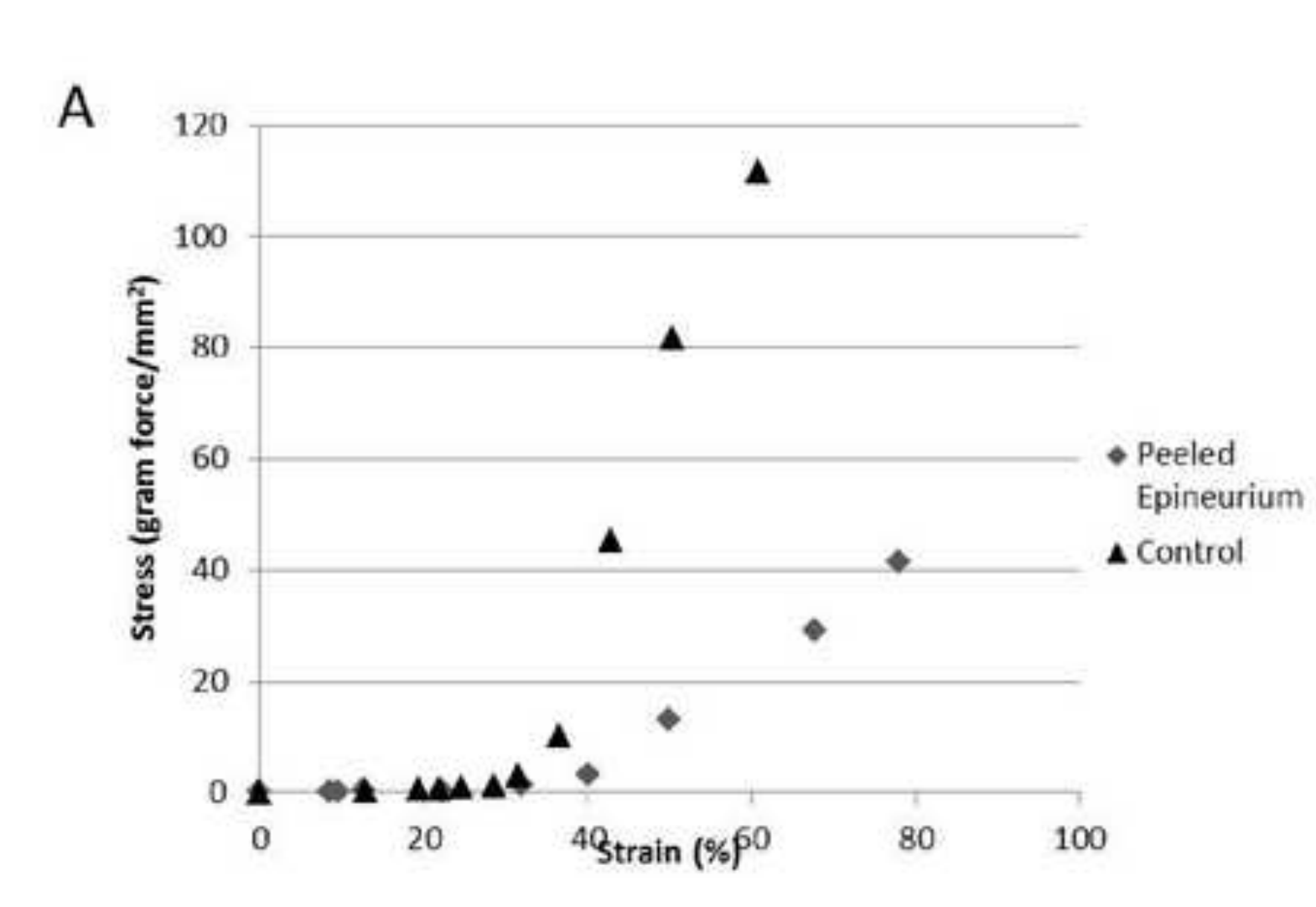

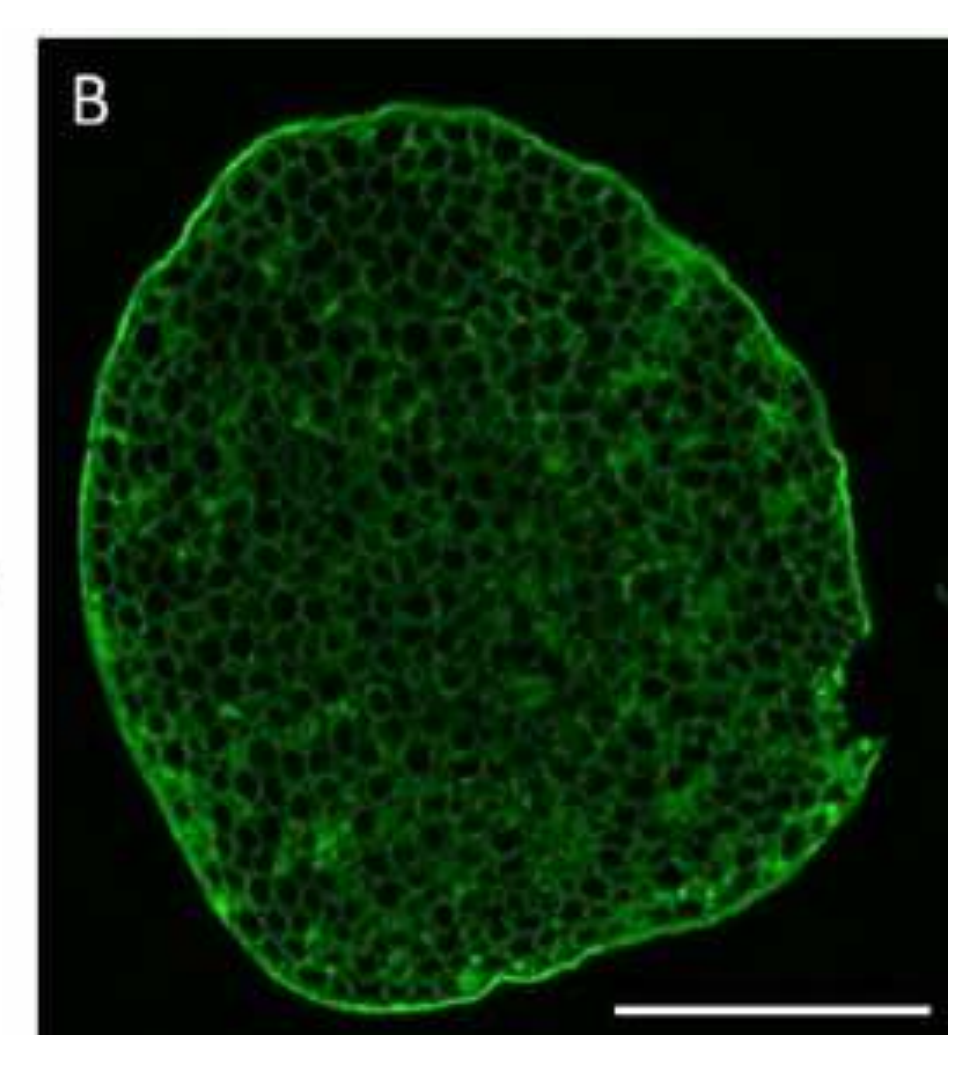




\section{A Cell Fraction Tensile Modulus After Paclitaxel Treatment}

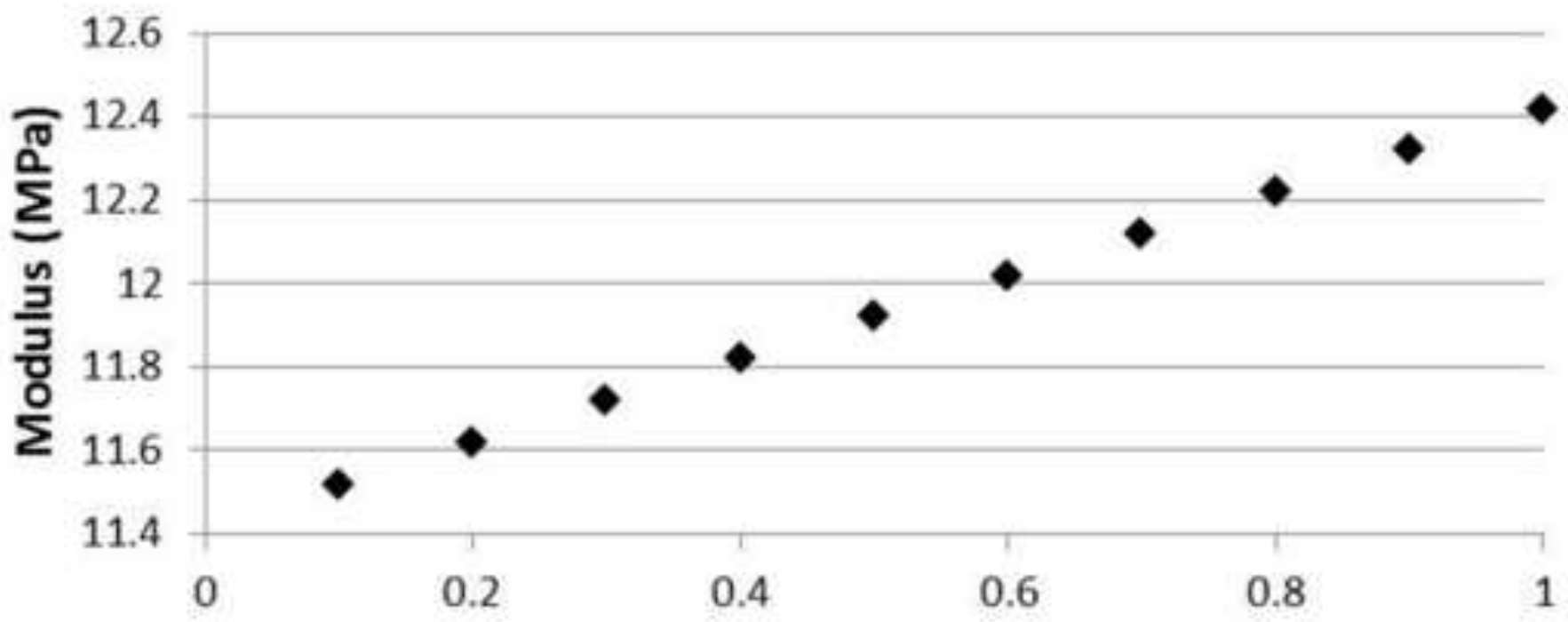

Initial Cell Fraction Tensile Modulus (MPa)

B

\section{Stiffening Factor}

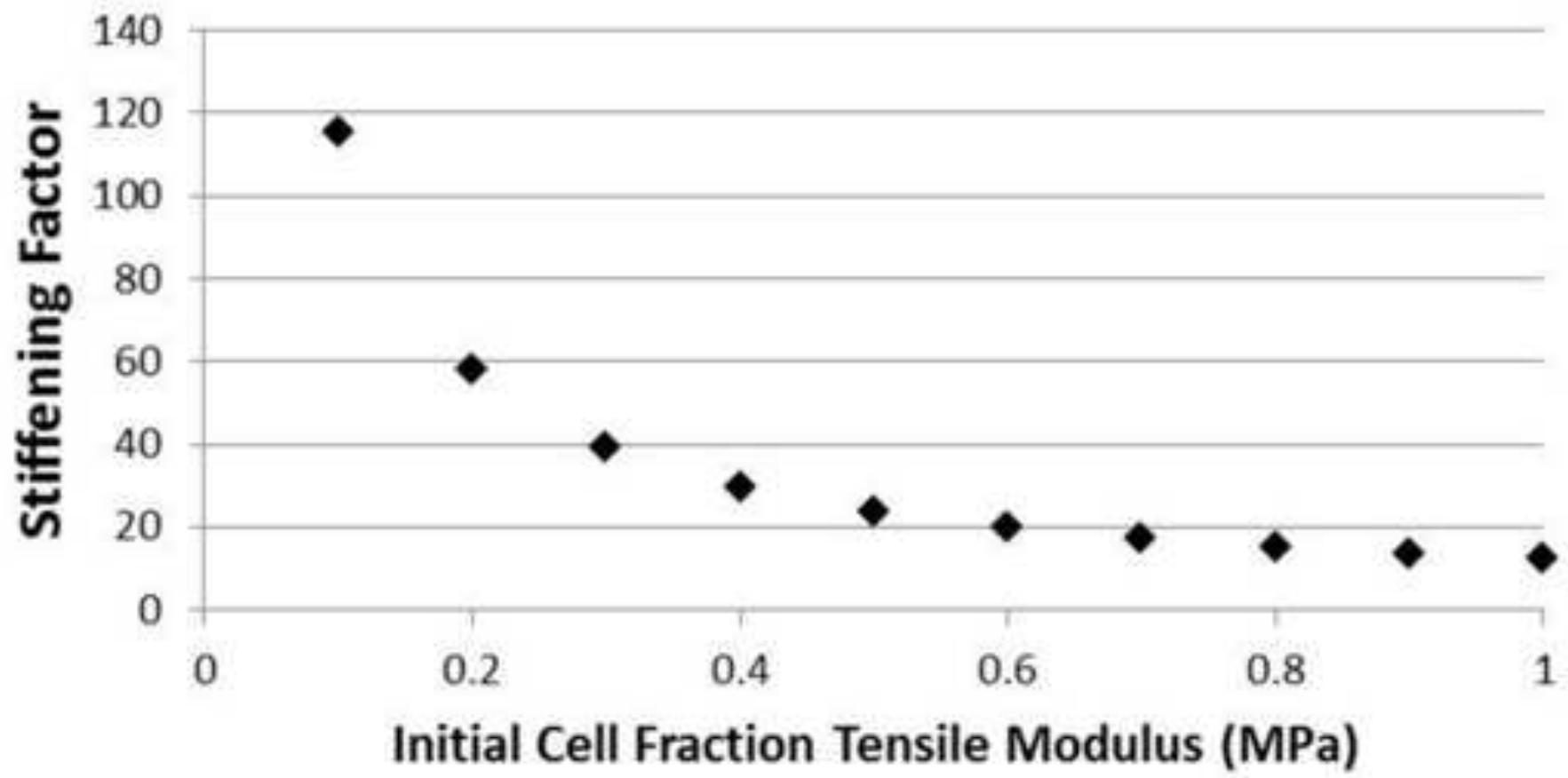

